vol. 33 - $n^{\circ} 2$ et $3 \mid 2017$

Dire la violence des frontières. Mises en mots de la migration vers l'Europe

\title{
«Tu ne pleures pas, tu suis Dieu... » Les aventuriers et le spectre de la mort
}

"You don't cry, you follow God..." Adventurers and the Specter of Death

"No lloras, sigues a Dios... " Los aventureros y el espectro de la muerte

\section{Cécile Canut}

\section{(2) OpenEdition}

Journals

\section{Édition électronique}

URL : https://journals.openedition.org/remi/8720

DOI : $10.4000 /$ remi. 8720

ISSN : $1777-5418$

Éditeur

Université de Poitiers

Édition imprimée

Date de publication : 1 septembre 2017

Pagination : 21-43

ISBN : 979-10-90426-59-7

ISSN : 0765-0752

Référence électronique

Cécile Canut, " «Tu ne pleures pas, tu suis Dieu... » Les aventuriers et le spectre de la mort », Revue européenne des migrations internationales [En ligne], vol. 33 - n² et 3 | 2017, mis en ligne le 01 septembre 2019, consulté le 16 avril 2022. URL : http://journals.openedition.org/remi/8720 ; DOI : https://doi.org/10.4000/remi.8720 


\section{"Tu ne pleures pas, tu suis Dieu... " Les aventuriers et le spectre de la mort}

\section{Cécile Canut ${ }^{1}$}

\section{Préambule}

Au large de Lampedusa. La caméra filme longuement les corps, inanimés, ces corps tirés dans le canot de sauvetage. Ces corps dont on ne sait pas s'ils sont morts ou vivants, que la mer mouvementée empêche de manipuler avec délicatesse. Ils sont extirpés de l'embarcation de fortune et entassés les uns sur les autres. Les membres se croisent, s'emmêlent, on entend respirer l'un, gémir un autre. II faut faire vite. On les retrouve quelques minutes plus tard, allongés, cette fois séparés les uns des autres sur le pont d'un bateau. À même le sol. Un médecin est en train de les ausculter. "Celui-ci est déshydraté ", d'autres sont intoxiqués par le pétrole qui a pénétré leur chair et brûlé leur peau.

Le naufrage a été terrible une fois encore. Quelques instants plus tard, le réalisateur, Gianfranco Rosi, s'oblige à entrer dans le fond de la cale où des dizaines de corps s'entremêlent, cette fois sans vie. On ne les distingue plus. Des bras, des jambes, des têtes émergent d'un amas de cadavres. Cet instant précis, le réalisateur nous a préparés à le voir sans fermer les yeux. Vient ensuite le silence des rescapés, assis à même le sol.

Une femme bredouille en faisant bouger sa tête et tout le haut de son corps, elle parle mais nous n'entendons pas bien ses paroles... Une autre, la tête penchée sur le bras de son amie, gémit. Son amie lui fait des signes apaisants tout en fixant un point devant elle.

Cette même femme revient peu après à l'image. Allongée par terre. Elle dit : "Où sont les Noirs? "On ne comprend pas. Parle-t-elle des morts? Elle s'assoit. Elle dodeline de la tête comme si aucune position ne pouvait lui convenir. Elle met sa tête en arrière. Elle implore quelqu'un. Mais personne ne peut lui répondre. Elle est seule face à un désastre dont on imagine qu'elle ne pourra jamais se remettre. Elle ne sait plus quoi faire de son corps, de ses mains, de ses yeux. Alors, elle prend la bouteille d'eau qui est devant elle et commence à la faire couler sur son visage. Et en ce court instant, elle semble s'apaiser enfin. Elle souffle, ferme les yeux. Elle vide toute la bouteille sur son visage, la tête rejetée en arrière.

\footnotetext{
1 Professeure des universités en Sciences du langage, Université Paris Descartes, CERLIS, 45 rue des Saints-Pères, 75006 Paris ; cecile.canut@ parisdescartes.fr Je remercie MahametTimera pour la lecture particulièrement pertinente de la première version de ce texte.
} 
Ce que le réalisateur de Fuccuoamare, Gianfranco Rosi, nous donne à voir dans la plus grande pudeur et le plus grand respect des personnes, c'est évidemment l'horreur de l'épreuve que subissent les rescapés des naufrages entre la Libye et I'Italie. Mais il tient à dépasser la fonction de témoin qui est celle du spectateur retenu par son admirable travail de la matière photographique et sonore. "Nous sommes tous, collectivement et individuellement, responsables de ces atrocités ${ }^{2}$ ", dit-il. La formulation est accusatoire, marquée du caractère impérieux qui est celui des verdicts, et cependant nous gagne à elle par l'émotion qui en est le canal. Associée aux images auxquelles elle donne suite, elle ne cesse de nous hanter.

\section{Introduction}

Face aux différentes mises en images, en textes ou en récits (littéraires, artistiques, cinématographiques) de la migration, toujours empreintes d'une dimension émotionnelle, la parole des voyageurs ${ }^{3}$, lorsque nous prenons le temps de l'entendre et de la réentendre, apparaît bien différente ${ }^{4}$. Au-delà du silence et de l'occultation des moments les plus difficiles, les récits des traversées parfois longs de plusieurs années, lorsqu'ils sont formulés (souvent longtemps après, donc avec beaucoup de recul) ne sont pas chargés de cette dimension tragique que nous ressentons à la vue des images des naufrages ou des traversées mortelles dans le désert. Si les femmes en parlent rarement, les hommes en disent un peu plus. Fatou, que j'aurai côtoyée avec ses deux enfants, pendant de longs mois dans le squat de Baudelique en 2009 (le " ministère de la régularisation de tous les sans-papiers " à l'initiative de la CSP75 $5^{5}$ ) me dévoile un jour dans la chambre que j'occupais avec elle, devant la caméra, qu'elle a fait la traversée en mer, tout en me demandant de n'en parler à personne :

" C : Mais Fatou tu savais que tu pouvais mourir? $\mathrm{F}$ : c'est sûr oui, c'est ça que je disais, là je raconte personne ça, j'étais FATIGUÉE. "

2 Gianfranco Rosi, entretien mené par Joseph Confavreux, [en ligne] consulté le 30/07/2016. URL : www.meteore-films.fr et http://www.rv-press.com/?p=3650

3 Concernant mon choix d'utiliser invariablement les termes d'aventuriers, voyageurs, refoulés ou exilés ou migrants, voir Canut, 2014b et 2016.

$4 C^{\prime}$ est un des résultats des travaux menés par le groupe Miprimo, La migration prise aux mots - projet de recherche (ANR) que j'ai dirigé entre 2010 et 2014 - autour des différents types de récits produits par les migrants eux-mêmes, sollicités ou non par les chercheurs. Cet article se fonde essentiellement sur les récits recueillis par mes soins au cours de longues ethnographies (approches anthropologiques) entre 2008 et 2013, au Mali et au Cap-Vert (ayant notamment donné lieu à trois films documentaires), mais aussi sur certains récits récoltés par l'équipe Miprimo au Sénégal, au Mali et au Cap-Vert, dont Caroline Panis, Véronique Petit, Aziz Faty, Daouda Gary Tounkara, Cheikhna Wague, Abdourahmane Seck. Y seront adjoints des extraits de récits recueillis par Gabriela Pujol (2011) étudiante de master sous ma direction ainsi que David Mahut, post-doctorant (Paris Descartes) récits issus, dans les deux cas d'ethnographies. Les transcriptions linguistiques (parfois traductions de langues africaines) ont été, pour plus de facilité de lecture, aménagées en transcriptions orthographiques assez classiques (la hauteur montante de la voix est indiquée par des majuscules, les rires par [***] et les allongements vocaliques par le signe [:]). Qu'ils soient filmés ou enregistrés par nos soins, I'ensemble des discours présentés sont oraux et résultent d'enquêtes ethnographiques, menées au Mali, au Sénégal, en France et au Cap-Vert avant, pendant ou après les trajectoires migratoires avec, dans la plupart des cas, des hommes bien plus que des femmes.

5 La CSP75, Coordination des Sans-Papiers de Paris (75), issue des luttes de 1996, dans I'église Saint-Bernard, regroupe plusieurs collectifs de Sans-Papiers (voir le site : https:// csp75.wordpress.com). 
Les moments où la mort surgit dans le voyage ne font pas souvent l'objet d'une narration détaillée, voire émue, qu'il s'agisse d'hommes ou de femmes: le registre émotionnel ou affectif est d'ordinaire absent de la narration. Tout peut porter à croire alors que la mort est chose admise. Voire que la pudeur cache une accoutumance en forme d'insensibilité chez les témoins autant que dans les familles qu'elle touche. Au point, pour certains, de se laisser aller à l'idée, profondément malthusienne, que la disparition des enfants, des frères et sœurs, et des plus jeunes en général, est inscrite dans le parcours de vie de chacun des natifs des régions à forte natalité, et qu'elle survient dans un amoindrissement du deuil et la résignation devant l'irréparable. C'est une conception des choses que plus de quinze années passées auprès de Maliens, de Sénégalais ou de Capverdiens notamment contredisent sans restriction ni réserve aucunes.

Il n'en demeure pas moins que les migrants occultent cette dimension alors même qu'ils cherchent une vie meilleure. De retour au pays, qu'ils aient réussi ou pas, ils racontent à leurs proches et construisent leur récit selon des perspectives et des visées qui leur sont particulières. Si chaque récit est singulier, la mort, elle, n'est généralement évoquée qu'à demi-mot, ou bien de manière à servir un but précis : s'insurger contre l'Europe ${ }^{6}$, critiquer les passeurs, obtenir les faveurs d'ONG qui les sollicitent (Maitilasso, 2014), etc. Tout se passe, au fond, comme si la mort, aussi proche et cruelle soit-elle, se constituait en un impossible de la narration envers les pairs : comme s'il fallait qu'elle soit inénarrable, ou indicible, et forme le point aveugle des discours de bon nombre de migrants.

Au-delà de l'étude des raisons sociales, politiques, économiques ${ }^{7}$ qui poussent les habitants d'Afrique de l'Ouest à partir, je propose de m'arrêter ici sur la dimension existentielle - puisqu'elle n'est pas autrement énoncée quand bien même elle fait ressortir des motifs à teneur religieuse ou métaphysique qui émerge des formes langagières utilisées par les aventuriers dans leurs récits ou discours, en me focalisant notamment sur les passages d'évocation de la mort. À travers la construction des récits, les modalités discursives ou les formes de dialogisme, j'examinerai de quelle manière la mort revêt presque systématiquement un caractère de subsidiarité dans le parcours mis en mots. Car si les aventuriers, dès le départ, usent de multiples formules du type "Barça ou Barsaaq " ("Barcelone ou la mort ") ou "Saya ka fisa ni maloya " (" La mort plutôt que la honte ") indiquant à quel point ils sont conscients du danger qu'ils encourent, ils ne sont nullement suicidaires. Ils ne subissent pas la situation, ils ne sont pas " prêts " à mourir pour aller en Europe. Au contraire, c'est animés d'une puissance de vie qu'ils construisent leur histoire, leur parcours et leur existence, qu'ils inventent de manière active leur devenir en se soumettant à l'épreuve des obstacles rencontrés. Dans cette quête, la place de Dieu comme garant d'un devenir devient alors essentielle : la mort d'un proche, à l'instar de l'image de Dieu, n'a pas à être évoquée, décrite, représentée dans les récits.

6 C'est ainsi que certains refoulés à Bamako, regroupés en association (ARACEM, Association des Refoulés d'Afrique Centrale au Mali, créée en 2011) ont décidé de faire de leur histoire une pièce de théâtre militante (Canut et Sow, 2014).

7 Cette dimension n'exclut pas ces aspects que j'ai déjà eu l'occasion de développer ailleurs (Canut, 2014b et 2013 ; Canut et Sow, 2014), ou qui ont été traités dans de nombreux travaux pionniers ('Quiminal, 1991 ; Clifford, 1997 ; Timera, 2002 ; Pian, 2009 ; Bredeloup, 2008 pour n'en citer que quelques-uns). 
Si elle doit advenir, c'est Dieu qui l'aura choisie, et ce choix est incontestable. Comme dit monsieur Fofana dans Frontière reconduite (Canut, 2008a) : "En tout cas la mort est entre les mains d'Allah je suis là ou là-bas je vais mourir, si cela se trouve que je meurs là-bas c'est Dieu qui le fait, c'est Dieu qui veut, c'est entre les mains de Dieu."

\section{" Je me suis levé pour quitter mon pays " : quand il faut partir...}

Badje vit à Doila, au Mali, où nous I'avons rencontré. II n'a aucune activité. II n'est pas marié. II n'attend que de repartir.

" Je me suis levé pour quitter mon pays, et partir à l'étranger. J'ai quitté Doila pour aller à Kayes, de Kayes à Nioro, puis jusqu'à Nouakchott... J'ai atteint Nouadibou. Et là, j'ai vu la mer. Une fois là-bas, on a cherché une pirogue, et surtout un moteur pour la pirogue. On s'est mis d'accord avec des gens. Ils nous ont emmenés. On est montés sur la mer, on a fait une nuit et un jour. Le deuxième jour, des gens sont arrivés derrière nous. Ils nous ont suivis. Ils nous ont attrapés sur la mer et ils nous ont ramenés. Ils nous ont ramenés en Mauritanie. Ils nous ont enfermés en prison. En prison, on a beaucoup souffert. On est restés en prison pendant un mois. Ils ne nous donnaient rien à manger. Ce qu'ils nous donnaient nous a rendus tous malades. Deux d'entre nous sont morts sous nos yeux. Deux autres sont morts derrière moi. " (Badje à Doila, Mali, dans Frontière reconduite, 2008, traduit du bambara par moi-même)

Dans ce récit de Badje, comme dans bien d'autres, I'aventure s'initie avec une action du corps, un mouvement, une mise en marche : "Je me suis levé [...]" Le départ prend consistance avec ce mouvement premier qui consiste à quitter la position assise ou couchée, synonyme pour les aventuriers d'impuissance, de honte et de dépendance, à la façon dont on se débarrasse d'un vieil habit, d'une ancienne peau - métaphore de l'habitude et de la soumission à des déterminations tout extérieures. Badje, jeune villageois qui a tenté - en vain - l'aventure pour la première fois, ne dit pas qu'il s'est levé pour faire sa valise, non plus qu'il s'est levé pour partir. La formule est des plus lapidaires et cependant le raccourci est saisissant : I'accompli de l'expatriation, tout exceptionnel qu'il soit, tient dans le geste inaugural du lever, qui est pourtant des plus communs. Car ce geste inaugural inclut déjà en lui tout le processus à venir, il a tout de la réponse à l'appel solennel entendu comme un commandement extérieur. L'action, entièrement englobée dans le simple geste, prend du coup une nouvelle signification et s'inscrit dans une perspective bien plus large, qui est le cadre d'un immense défi. Car s'il y a bien sûr une espérance, il y a aussi une culpabilité sous-jacente dans le fait de quitter son pays : on abandonne les siens, mais surtout on rompt un destin premier, celui que Dieu avait voulu pour chacun, en le faisant naître à tel endroit parmi tels gens.

Les personnes qui partent, dès qu'elles partent et comme elles partent, sont partagées entre un espoir un peu fou, hors-norme, et une crainte à la mesure de cet espoir, qu'elle vient contrebalancer, dont elle est la part sombre à surmonter. Sortir de son pays, quitter les siens, n'est pas une mince affaire, quand bien même le départ peut être instigué par eux-mêmes. Il faut à ce départ des raisons impérieuses, des causes habitées d'une force à laquelle rien ou presque ne 
pourrait s'opposer.

Ces raisons impérieuses sont d'abord celles du corps : Ia faim ou la menace de la famine sont souvent évoquées ; ces raisons sont sociales, et plus largement économiques :

"Il faut bien manger, il faut bien s'habiller, il faut acheter une maison, il faut se marier, il faut acheter une voiture. C'est ça, c'est ça qui fait que les jeunes sortent : mais s'il y avait du boulot ici personne n'allait sortir. C'est par manque de moyens, c'est vrai, ces moyens-là il faut les chercher. " (Aliou Boubou $\mathrm{Ba}$, propos recueilli en français, par Aziz Faty à Boynadji dans la région de Matam, Sénégal, en 2012)

Pour autant, l'argument économique en lui-même ne suffirait pas à justifier pleinement le départ, et pourrait bien se voir opposer le reproche de l'individualisme. Car ce serait une faute majeure que de partir à titre personnel : celle de l'abandon de ses proches, c'est-à-dire celle de l'orgueil et de l'envie, celle du trop grand amour de soi. Si ce désir d'autonomie et de liberté régit les pratiques de bien des aventuriers (Timera, 2002) il n'est guère avouable comme tel. À telle enseigne qu'il est alors précisé que l'argument économique est surdéterminé par celui du dévouement, sinon du sacrifice : cette misère n'est pas d'abord la sienne, mais celle des parents, de la famille - elle est le malheur de ceux auxquels on se doit de porter secours et assistance :

"Ton père n'a rien, ta mère n'a rien, toi-même tu n'as rien. On s'assoit et on se regarde les uns les autres... " (Mariko et Fomba, avec Koyan à Doila, Mali, dans Frontière reconduite, 2008, traduit du bambara par moi-même)

Issu du monde paysan Aliou, tout comme les deux frères Mariko et Fomba, insiste sur l'obligation au départ, le non-choix :

" Non non, moi franchement parler, l'aventure ne m'avait pas tenté, l'immigration ne m'avait pas tenté, moi si mes parents avaient eu les moyens et encore de m'aider pour vraiment étudier quoi disons je n'aurais jamais bougé du Sénégal. Franchement parler, et si je suis sorti c'est tout juste pour leur venir en aide [...] et j'ai des parents pauvres, donc j'ai été obligé d'aller à l'aventure pour au moins pouvoir leur venir en aide."

(Aliou Boubou $\mathrm{Ba}$, propos recueilli en français, par Aziz Faty à Boynadji dans la région de Matam, Sénégal, en 2012)

C'est souvent poussé par une nécessité qui dépasse leur propre personne que certains aventuriers se mettent donc en route. Car s'il ne s'agit pas d'un coup de tête, il ne s'agit pas exactement non plus d'une véritable décision, en tout cas pas de la décision d'un projet formé autour d'objectifs individuels à atteindre : quitter son pays et les siens ne constitue pas un plan de vie personnel. C'est bien plutôt d'une volonté qu'il est question, une grande volonté, entendue comme extérieure, adventice : une force ne procédant pas exactement d'un choix personnel, aussi difficile soit-il. II n'y a rien, ici, de l'affirmation d'un Moi désirant ce qui n'empêche bien entendu pas, par la suite et afin de relever cet immense défi, un engagement individuel sans relâche.

Le champ lexical de l'obligation, du devoir, de la nécessité revient dans presque tous les récits. II " faut " s'en aller, il " faut " bouger, il " faut " sortir. $C^{\prime}$ est presque contre lui que le migrant part : l'individu souverain n'est pas 
représenté au cœur du processus. Sa volonté de migrer se présente à l'inverse comme une sorte d'aboutissement, la suite logique donnée à un enchaînement de causalités qui en font le débiteur de ses proches : la dette contractée notamment vis-à-vis des anciens, de sa femme, et des plus jeunes autour de lui, constitue, d'un bout à l'autre, un moteur du processus aventurier :

"Moi, je n'ai jamais songé à faire l'aventure, même quand j'étais petit, mais quand tu vois ton père et ta mère, tu vois ta marmite comme ça qui est au paradis, inclinée, qui est au paradis, parce que la marmite veut toujours aller à l'enfer..." (Aliou Boubou Ba, propos recueilli en français, par Aziz Faty à Boynadji dans la région de Matam, Sénégal, en 2012)

Il est dit par-là que la part de soi dans le processus initial est souvent congrue, réduite au minimum. Le corps qui se lève pour quitter le pays n'est qu'à peine mu par sa personne, et la décision malgré tout prise de se mouvoir est comme un point d'incandescence de la conscience soumise à la rigueur des faits. Les aventuriers sont au plus haut point partagés : pris entre leur devoir de soutien familial et la nécessité du départ ; pris entre l'aspiration à l'ailleurs et l'assentiment au natal enjoignant d'endurer son sort présent ; pris entre la fascination qu'exercent les fastes européens et la fidélité due à ses ascendances. II s'ensuit, dans cette opposition de forces irréductibles, des tiraillements comme une culpabilité qui font tout l'inconfort de certains migrants saisis dans leur intimité :

" Mais vraiment, VRAIMENT ! Bon sur ma part, bon, je regrette quoi, pourquoi j'ai sorti mon pays ? Parfois ça me fait mal, MAL, pourquoi Dieu m'a, m'a donné cette chance-là quoi, pour sortir mon pays ? Ça me fait MAL, j'ai un GRAND REGRET, vraiment, mais, n'a pas le choix, parce que quand tu penses, si j'étais là-bas peut-être, tu es à côté de ta FAMILLE. C'est MIEUX quoi, tu vois ta famille, tu souffres pas beaucoup. Mais, tu te sens quelque chose, y a quelque chose qui manque dans la FAMILLE quoi. C'est pourquoi bon, on est obligés de sortir comme ça, bon, mais c'est PAS MON CHOIX, vraiment, je n'aime pas, je n'aime pas, je n'aime pas, je suis sorti mais, je n'aime pas. " (Harry, Sénégalais, propos recueilli en français, par Caroline Panis à Sal, Cap-Vert, en 2012)

Comme d'autres, coincés sur l'île de Sal où il a fini par s'installer, Harry est partagé, en perpétuel équilibre (ou plus exactement une suite continue de déséquilibres enchâssés) entre l'exigence de soumission à la grande volonté, qui l'a poussé à quitter son pays, sa famille et son toit, et l'affirmation de sa personne en une forme sobre ou pudique d'héroïsation. Car même lorsque le but est atteint, une fois en France, certains ressentent le même inconfort soutenu par la même exigence de responsabilité envers les siens :

"On veut aller jusqu'au bout, on ne s'arrête pas, parce que on peut pas vivre dans la pauvreté. On peut pas vivre dans la pauvreté : peut-être t'as t'as des femmes, t'as des enfants, la famille sur le dos, t'as rien, qu'est-ce que tu vas faire? Ben, rester il faut pas rester là-bas tu bouges, tu vas aller chercher là où tu peux trouver, nous on va sortir, puis on envoie à la famille, la nourriture, des trucs comme ça, c'est pour ça que tout le monde est là hein ? [...] On n'est pas là pour, dire voilà, la France, il y a des gratte-ciel, les les choses comme ça on est pas là pour ça. On est venus chercher du travail, avoir des sous, servir la famille, avoir un petit papier. Tu viens un an, TU VAS ET TU VIENS TU VAS ET TU VIENS. " (Dja, Malien, propos recueilli en français par Gabriela Pujol à Paris - Pujol, 2011) 
Ce va-et-vient, ce mouvement pendulaire devenu un inaccessible de la migration en contexte de fermeture des frontières, est avant tout celui des sentiments qu'inspire le grand départ ainsi que ce jeune père l'exprime ici : ce qui va et vient n'est pas tant l'individu lui-même que ce qu'il éprouve et ressent au long de l'aventure et ce balancement est l'expression d'un partage difficile, et même, par bien des aspects, insurmontable. II se matérialise par un quotidien souvent totalement pris entre ces deux modèles inconciliables : les repas, les musiques, les conversations, les manières d'être et de faire du pays qu'on a laissé, d'un côté, et, de l'autre, la vie administrative, professionnelle, relationnelle qu'impose le séjour en Europe.

Les contradictions inhérentes à la situation sont partout présentes : c'est le propre de la parole que de les faire émerger. Elles affleurent en chacun des discours tenus. Elles s'expriment même à travers le seul fait de narrer sa propre aventure et d'en confier les ressorts presque intimes en les plaçant sous le signe d'une volonté supérieure. En effet, la tentation est grande, a fortiori chez les refoulés mortifiés par leur échec, de se dire revenant, donc faire le récit, d'une expérience inénarrable - une épreuve surmontée, au moins en partie, et conférant à son protagoniste une part d'héroïsme ou tout du moins la vertu de ceux qui acceptent le verdict du destin et n'en sont pas moins enclins à en forcer à nouveau le cours : ceux que l'humilité, d'un côté, pousse en lisière de l'humiliation, qui les oblige à la résipiscence, et que par ailleurs la bravoure porte à imiter les anciens, leurs frères.

L'appel, pour certains, est renforcé par l'exemplarité d'autrui (Jonsson, 2007 ; Bredeloup, 2014), par le désir d'imiter ceux qui ont réussi, ceux qui racontent l'ailleurs et appellent leurs frères. Dans les récits, ces voix venues d'ailleurs, véritables leitmotive, apparaissent comme des catalyseurs de la " sortie ". Elles obligent à partir :

"Ouais c'est la famille, certains c'est la famille, certains c'est des voisins certains, bon. En voyant ça, on se disait ben : voilà *** là-bas c'est c'est, donc faut y aller quoi. Et tout le monde disait qu'est-ce que tu vas attendre il faut : et voilà on attend plus. " (Abdoulaye, Malien, propos recueilli en français avec Gabriela Pujol à Paris - Pujol, 2011)

Abdoulaye, comme bien d'autres, décrit les interactions langagières qui président au départ : d'une part ceux qui ont réussi (les voisins, les autres) et qui suscitent le désir de départ, d'autre part la voix des proches (" tout le monde ", " la famille ") qui poussent à l'action. Les formes polyphoniques ou dialogiques (Bakhtine, 1952), par lesquelles les paroles d'autrui se trouvent intriquées dans les récits, sont déterminantes pour comprendre le processus singulier de la mise en marche qui advient comme malgré soi. César (refoulé camerounais à Bamako, devenu responsable de l'ARACEM ${ }^{8}$ à Bamako) insiste sur ces tiers extérieurs, en les nommant et en citant directement leurs paroles :

"Un jour, on m'a appelé pour m'annoncer qu'André, un garçon que j'avais rencontré à l'université de Tamanrasset, était passé en Espagne. Je l'ai appelé et il m'a dit: "Boxeur, qu'est-ce que tu fais là ? Il faut venir ici, c'est mieux. Si tu boxes en Espagne, c'est sûr que tu pourras aller plus loin. Laisse tomber l'histoire de l'Algérie, c'est toujours l'Afrique,

8 Voir note 6. 
c'est toujours les frères !" [...] Mourad, le journaliste, m'a demandé de rester mais je lui ai dit non, je m'en vais. C'est comme ça que j'ai pris la route. " (César, récit recueilli en français par Martine Blanchard à Bamako, Mali, en 2008)

Non moins que les représentations visuelles (télévision, internet) dont les effets sont puissants - c'est-à-dire propres à magnétiser des esprits que fascine la possibilité d'un arrachement à l'indigence et à la monotonie -, les récits des migrants arrivés en Europe, donnant des informations par téléphone, ou des revenants, les " migrants de retour " arborant leur réussite, sont déterminants dans ce qui s'impose comme une nécessité : aller voir, prouver que l'on est capable d'y aller et de réussir. Autrement dit, de se montrer l'égal de ses prédécesseurs, de rejoindre les rangs des glorieux aînés. Autrement dit encore, de se hisser aux côtés de ceux-là et se trouver dès lors à la mesure d'un devenir qui, au départ, ne connaissait pas de mesure et tutoyait le surnaturel. Dans ce rapport au surnaturel, au tragique comme au miraculeux, la mort tient immanquablement une place, fût-ce sous l'aspect dénégatoire : par le silence fait sur elle, ou par la marginalisation - feinte, ou détournée - de ses effets sur les esprits.

\section{L'incidence de la mort : " Vous l'enterrez rapidement... "}

Tout au long de leur trajectoire, souvent interminable et qui tend à revêtir parfois un caractère cosmique, souvent composée de longues pauses en chemin (Timara, 2009), les aventuriers sont amenés à croiser la mort. Mais pour autant qu'ils puissent en redouter la survenue, elle ne vaut pas condamnation de l'entreprise.

« C : Mais, tu aurais pu mourir. Tu aurais pu mourir dans la mer... B : Hein? Oui!

C : Tu n'as pas peur? B : Non.

C: Pourquoi?

B : Pourquoi ? Parce que la mort est moins terrible que la honte. La mort vaut mieux que la v... que la honte. Tu as tes pères, ils n'ont rien, ils n'ont même pas de quoi manger. Comment tu peux trouver le sommeil ? L'endroit où il est facile de trouver de l'argent, c'est là que tu dois partir le chercher. Mourir dans la maison, c'est une chose qui est grave, mais si tu meurs alors que tu es parti chercher de l'argent, pour moi, ce n'est pas une mauvaise chose. Donc si tu me demandes, vraiment, encore aujourd'hui, si je retrouve la santé, je suis prêt à repartir... jour et nuit. " (Badje à Doila, Mali, dans Frontière reconduite, 2008, traduit du bambara par moi-même)

Le regard de Badje est tout autant déterminé que sa voix. La mort ne l'arrêtera pas. La mort est toujours sur la route : fatigue, déshydratation, maladie, malnutrition, violence aux frontières, conflits internes, noyade, etc., la longue liste des obstacles fatals a été maintes fois relatée (Andersson, 2014 ; Kobelinsky, 2016, Choplin et Lombard, 2010 ; Khosravi, 2007 ; Pian, 2009). Or, elle n'apparaît que rarement au centre des récits de migration, excepté lorsqu'un chercheur $s^{\prime} y$ intéresse : elle intervient par incidence, indirectement, et ne se présente qu'en dommage collatéral inhérent à l'aventure, et non en tant que dénouement paroxystique d'une épreuve longue et difficile. Dédramatisée, elle ôte à cette 
aventure, même interrompue de la pire des manières, un caractère tragique. Un réel vitalisme, aussi assombri qu'il puisse être par la disparition de bon nombre d'entre eux, anime les aventuriers. C'est ainsi que Mariko et Fomba, les deux frères, l'évoquent comme un fait supposément bénin au beau milieu de notre discussion sur leur parcours :

" M : Imagine : ton petit frère, ton petit frère, même père, même mère. Vous partez ensemble faire la route et à un moment donné, il ne peut plus avancer. II s'assoit. Tu l'encourages pour qu'il se relève, il ne peut plus se relever. C'est ton frère de lait mais tu vois que tu n'as pas de solutions. II meurt dans tes bras. Ou bien, il s'assoit alors que les autres partent, et tu es obligé de les suivre. Tu n'as pas le choix.

II meurt, assis là-bas. Tu n'as pas le choix, tu l'abandonnes.

$\mathrm{F}$ : Souvent, s'il meurt, vous le recouvrez de sable rapidement et vous partez vite fait. Vous l'enterrez rapidement. $\mathrm{M}$ : Même si c'est ton frère.

$\mathrm{F}$ : Eh, mais tu ne peux plus retourner en arrière. Lui, il ne fait plus partie du groupe.

$\mathrm{M}:$ Est-ce que tu peux retourner en arrière?

Toi-même tu vois que tu n'as pas d'autres solutions.

$\mathrm{F}:$ Il ne peut pas survivre parce que tu ne peux rien faire pour le dépanner. $\mathrm{M}$ : S'il meurt seulement, vous le laissez là. Vous le couvrez de sable. Et vous continuez. Vous continuez ainsi, puis un autre aussi échoue. II va dire: "Je ne peux plus continuer, je reste assis là, je vais mourir ici." Vous lui dites: "On doit continuer la route."

$\mathrm{F}:$ On le laisse là, dans la brousse.

$\mathrm{M}:$ On continue petit à petit, les plus jeunes, eux, vont doucement. Certains vont jusqu'au bout, d'autres retournent. Mais en tout cas, tu fais plus de $1000 \mathrm{~km}$ à pied pour y arriver. "

(Mariko et Fomba à Doila, Mali, dans Frontière reconduite, 2008, traduit du bambara par moi-même)

En faisant appel à l'imaginaire de leurs interlocutrices (Koyan, mon amie malienne qui participe aux discussions, et moi-même), Mariko et Fomba construisent ce passage narratif sous une forme impersonnelle en utilisant le présent et le " tu " générique permettant d'une part de s'extraire émotionnellement de la description de la scène, et d'autre part d'impliquer l'auditoire en le plaçant au cœur de la situation. L'ouverture par l'impératif « imagine " suppose de se représenter un événement inimaginable qui va pourtant se produire comme une scène banale de la vie quotidienne d'un migrant et à laquelle il faut s'habituer.

L'interaction entre les deux frères s'imbrique au point de former un récit continu au sein duquel leurs interventions se répondent et s'enchâssent. Alors qu'ils évoquent une situation douloureuse à laquelle la migration peut conduire, celle de l'abandon d'un frère de lait dans le désert, ils tentent en même temps de se justifier en suscitant notre réflexion et notre compréhension. Mariko pose une question qui pourrait être la nôtre : " Est-ce que tu peux retourner en arrière ? " et y répond lui-même : "Toi-même tu vois que tu n'as pas d'autres solutions. "La situation (comparable à des expériences extrêmes d'exode forcé ou de guerre par exemple) est sans recours, il n'y a pas de place pour le contingent : pas de place pour l'atermoiement, pour un quelconque pathos, c'est-à-dire en somme pas pour l'expression d'une conscience émue. Le temps et l'espace se resserrent au point de ne former qu'une ligne, une ligne de fuite : tout est de l'ordre de la nécessité. Les modalités du devoir et du pouvoir (ou de 
l'impouvoir) se succèdent : "On doit continuer ", "Tu ne peux rien faire ", etc. Les assertions impératives sont de mise : "Vous le laissez ", "Vous le couvrez ", "Vous lui dites ", "On le laisse ", etc. Les conditions commandent, et cette impossibilité de choix du migrant, acculé aux pires actes de la vie, est souvent dévoilée longtemps après le trajet. Comme si s'ajoutait à l'épreuve de la mort, l'épreuve des larmes interdites et du deuil retenu - le temps long de la douleur endurée, doublement endurée parce que muette. Les modalités énonciatives et la co-construction discursive indiquent ici à quel point la question de l'émotion en relation à la mort (tel que je la rends tangible par mes questions) n'a pas sa place ici puisqu'elle ne dépend plus du tout de soi, puisqu'il n'est strictement pas possible d'en empêcher le cours.

Un autre type d'évocation de la mort, guerrière cette fois, est mise en récit par César au moyen du " on " ou du " nous ", soit un groupe d'aventuriers qui part à l'attaque de la barrière de Melilla (en 2005). Les modalités du devoir restent toujours très présentes:

" On avait attaqué la barrière trois fois en une semaine, trois fois en une semaine! La semaine suivante on a décidé de faire une attaque générale. Je faisais partie du comité d'organisation. [...] On allait utiliser la force pour attaquer la grille et on allait faire le Hoha. Le Hoha, ça veut dire que ce soit la vie ou la mort, il faut traverser.

On était prêts à risquer de mourir pour passer de l'autre côté. [...]

Le matin, on est descendu en convoi, on était plus de mille. Quand on est arrivés au goudron, les forces marocaines qu'on appelait les Ali nous attendaient. Ils ont commencé à tirer avec des armes à blanc qui font beaucoup de bruit, pour nous faire reculer. Qu'est-ce qu'il fallait faire? Nous avons compris que nous avions été trahis. Parmi nous il devait y avoir des traîtres qui avaient été payés. Nous étions une foule si nombreuse que nous ne pouvions plus rentrer dans la forêt. Qu'est-ce qu'il fallait faire? Attaquer! Quoi qu'il devait se passer! C'était le Hoha : même s'il faut que des gens meurent, il faut que les autres traversent. II fallait atteindre la grille à tout prix, ne pas retourner quoi qu'il arrive! Nous étions à cinq cents mètres de la grille, il fallait avancer, ne pas revenir en arrière. Nous avions instauré une loi : celui qui retournait à la forêt serait sanctionné. Tout le monde en avant, quoi qu'il arrive ! " (César, refoulé camerounais au Mali, récit recueilli en français par Martine Blanchard à Bamako, Mali, en 2008).

Fofana, refoulé lui aussi en 2005 de Melilla, probablement le même jour que César, fait part de sa rage devant les injustices et les aberrations de la politique migratoire dont lui et ses amis ont eu à subir les méfaits :

"Quand on a grimpé sur le grillage, ils sont venus, ils nous ont dit de nous arrêter. Tu comprends? Bon, certains se sont arrêtés, d'autres ont continué, comme nous étions très nombreux. On a voulu forcer pour faire tomber le grillage et le grillage est tombé. Ils sont arrivés, certains avec des pistolets, certains avec des matraques pour taper tout le monde. J'avais un ami, un Camerounais, ils lui ont dit de s'arrêter, il n'a pas voulu, ils ont tiré sur lui. Un autre, un Guinéen, ils lui ont tiré dessus aussi. Un autre, un Malien surnommé Rougeot, lui aussi, ils lui ont tiré dessus.

9 Épisode de sa vie qu'il mettra en scène plus tard avec ses camarades, dans la pièce Essingan, créée à Bamako en 2008 lors du Forum de l'AME (Association Malienne des Expulsés). Voir Canut et Sow, 2014. 
Après avoir tiré dessus, ils les ont mis dans une ambulance. Ensuite, dans la prison, ils sont venus avec les photos : de leur cadavre. Il y avait sept morts. Ils ont demandé si on les connaissait. Moi, j'en connaissais trois. Il y avait deux Maliens et... Non. Un Malien, un Camerounais et un Guinéen. Je les ai reconnus. Mais pourquoi ils les ont tués ? Parce qu'on se rend chez eux ? C'est parce qu'on va chez eux ? C'est ça ? Mais si on va chez eux, ce n'est pas pour violer leurs femmes. Ce n'est pas pour voler, ce n'est pas pour tuer des gens. C'est pour chercher du travail. Hein? Pour chercher du travail et de l'argent. Hein ?" (Fofana à Bamako, Mali, dans Frontière reconduite, 2008, en français)

Les questions retournées à l'auditeur, de manière rhétorique puisque Fofana n'attend pas de réponse de ma part mais répond lui-même de manière véhémente à ses propres questions, laissent pointer une colère et une révolte chez le jeune homme qui a perdu ses amis et s'est de plus fait expulser. II s'indigne de l'injustice de ces morts, de la disproportion d'une réponse politique à un simple besoin de survie économique. Et pourtant, comme tous, il ne chercher qu'à repartir.

À plusieurs reprises, César, dans le récit très détaillé de son voyage, insiste sur la nécessité de se comporter en guerrier, de ne jamais perdre le moral, d'aller de I'avant, de " se battre contre soi-même ". Perdu dans le désert, il raconte comment il motive son groupe en faisant fi de la mort : le récit implique de fait une occultation de toute affectivité. Le parcours nécessite de faire abstraction de ses émotions, de ses sentiments ("II fallait avancer, ne pas revenir en arrière "), attitude que l'on retrouve plus tard, en exil, lorsque la coupure avec la famille s'impose pour éviter la nostalgie. Cette éthique du combat, du migrant combattant qui doit tenir l'idée de la mort à distance et ne pas s'en trouver affecté, revient dans les récits de multiples manières. Ainsi, Harry, comme Jules à Paris, ou Souleymane au Cap-Vert, répètent qu'il " ne faut jamais baisser les bras " :

"Il faut se battre pour survivre, c'est ça l'Afrique, il faut se battre pour survivre. " (Harry à Sal, Cap-Vert, propos recueilli en français par Caroline Panis, en 2012)

L'éthique du combat implique alors de faire silence sur la mort ou de ne l'évoquer que pour convenir de son indifférence aux êtres, de sa froideur toute naturelle, en dehors même de ses actualisations les plus atroces, ce n'est pas le signe d'un oubli. Tout à l'inverse : c'est en creux, au creux de ce silence qui est aussi le sien, que la mort se fait présente. S'il est si souvent fait silence sur elle, c'est aussi qu'elle-même fait silence sur tout ce qu'elle atteint. La mort terrifie, et c'est peut-être bien une façon d'en conjurer l'effroi, pour le plus grand nombre, que de ne pas en parler. C'est la tenir à distance que de ne pas s'en émouvoir outre mesure. C'est comme la dompter que de ne lui accorder qu'un regard neutre ou dépassionné, voire de ne pas même lui prêter consistance à l'aide de mots : ne pas lui offrir ce linéament, ce début de visage, ce premier commencement de présence que lui octroie la parole.

II n'est pas insensé de penser qu'entre une part de superstition dans ce refus de prendre aux mots la mort. La mort fait peur, c'est l'évidence, ne serait-ce qu'à lever un coin de voile sur sa réalité. N'est-ce pas la fréquenter que de trop s'attarder sur une tombe ? N'est-ce pas déjà l'inviter à venir que de trop en parler? N'est-ce pas attirer sur soi le malheur que de prononcer son nom ? Et c'est ainsi le même Fofana qui, après s'être insurgé contre les meurtres commis par les gardes-frontières, confesse, d'une voix basse : 
"En tout cas la mort est entre les mains d'Allah, je suis là ou là-bas, je vais mourir, si cela se trouve que je meure là-bas c'est Dieu qui le fait, c'est Dieu qui veut, c'est entre les mains de Dieu. " (Fofana à Bamako, Mali, dans Frontière reconduite, 2008, en français)

\section{«Tu suis Dieu... Tu t'endurcis »}

Au moment où il se lève pour quitter son pays, Badje, le jeune homme rencontré à Doila, se montre animé d'une volonté dont la puissance semble excéder celle de sa seule personne - une volonté redoublée, transformatrice autant qu'elle-même transformée, par la dimension extraordinaire de l'enjeu : rompre avec une existence toute tracée. Mais s'il y a quelque chose d'héroïque dans l'aventure, cet héroïsme risque de se voir battu en brèche, jusqu'à laisser la part d'impudence et d'orgueil qu'il supposait l'emporter sur tout autre motif tant le parcours tient du calvaire : les drames se succèdent, et c'est poreuse ou floue qu'apparaît alors la frontière entre la bravoure et la divagation, faisant insensiblement passer de I'héroïsme à la déraison. Car si " c'est Dieu qui veut " comme dit Fofana, alors il n'est pas aberrant de penser que la vie toute tracée qu'il a voulu quitter se devait d'être acceptée comme telle : ce destin-là était peut-être le bon, et lui contrevenir aura non plus seulement tenu de la rupture, mais aussi de l'impudence et de l'affront. Le destin, tel que Fofana et d'autres en appellent à lui, pouvait être aussi bien celui que sa naissance, sous le jugement de Dieu, annonçait : celui d'un villageois laborieux. Le destin de l'aventurier est celui qu'il se choisit, du fait de la décision qu'il prend de partir et de rompre avec les siens. Quand bien même il se replace alors à nouveau dans l'aventure sous le jugement de Dieu, sa décision introduit la question de sa responsabilité. La morale, ou le rapport à la loi, la loi supérieure, entrent alors en jeu. Ata Koko, ce jeune homme parti du Togo pour arriver au Sénégal, a traversé bien des frontières, mais il donne un sens à chacun de ses actes ${ }^{10}$ :

"Ce n'est pas la chance, moi chez moi la chance n'existe pas, moi c'est Dieu qui donne, voilà, ce qui doit arriver Dieu l'a déjà programmé. "

(Atta Koko à Dakar, Sénégal, propos recueilli en français par moi-même)

Si une constante traverse tous les récits, il s'agit bien de l'importance accordée au rôle de Dieu dans le périple, ainsi que Timera (2009) a pu le souligner ${ }^{11}$. Pas un aventurier $n^{\prime} y$ échappe, ne serait-ce qu'en ponctuant son récit d'expressions idiomatiques: "Al'hamdoullilah, Dieu merci ", "Inchallah ", " S'il plaît à Dieu ", "Mais Dieu a fait ça ", "Dieu nous a donné ça ", "Dieu seul sait ", etc. Si la chance est un élément constant qui doit accompagner l'aventurier, celle-ci est toujours sujette à la volonté de Dieu qui leur permet de tout accepter, même le pire.

10 Voir notre article consacré à son imaginaire migratoire (Canut, 2013).

11 « Pour ces migrants, la souffrance, voire le compagnonnage avec la mort, s'insère dans une destinée sociale dont le récit mobilise des registres religieux et moraux qui énoncent l'impossibilité de se soustraire à la volonté divine, affirment que l'enfant mâle doit connaître la peine et envisagent l'épreuve de la souffrance comme une voie pour "devenir quelqu'un". La réalisation de cette destinée repose sur une volonté et une forte implication individuelles et, en même temps, ses issues sont acceptées avec humilité comme relevant de la volonté divine. " (Timera, 2009) 
"J'essayais de remonter le moral aux gars en leur disant: "N'ayez pas peur, allons-y les gars! Rien ne peut nous arriver ! On ne connaît pas le désert mais Dieu existe !" "

(César au Mali, récit recueilli en français par Martine Blanchard, en 2008)

Qu'ils soient chrétiens ou musulmans (tous nos interlocuteurs se réclament d'une religion monothéiste), les voyageurs ne cessent de l'invoquer :

"Mais si tu as la chance grâce à Dieu de... Mais tu demandes juste au bon Dieu de te donner la force de les oublier le temps de sortir de cette impasse. " (Amadou kane,

Fouta, propos traduit du poulaar par Aziz Faty à Dakar, Sénégal, en 2012)

"En Côte-d'lvoire quand j'allais, je n'avais aucun parent sur place. Dieu seul était mon unique recours. " (Mouhamadou B., propos recueilli et traduit du wolof par Abdourahmane Seck à Dakar, Sénégal, en 2012)

"Nous demandons au Bon Dieu de nous faciliter ce que nous souhaitons tous, qui est la route. Qu'Allah nous l'accorde. [...] Il y en a ici qui n'ont pas de papiers, mais c'est

Dieu qui a le papier à quelqu'un. Parce qu'il y en a qui sont partis, mais qui ne s'attendaient pas à partir. Il y en a même qui sont partis qui n'avaient pas de papiers. "

(Discussion collective avec Lassana S. et Samba Lassana C. recueillie et traduite du soninké par Cheikhna Wague à Dakar, Sénégal, en 2011)

Lorsqu'enfin la chance sourit, alors on remercie Dieu :

"Et si ça marche, on va dire : "Dieu merci !... II a fait quelque chose !" " (Dramane à Doila, Mali, dans Frontière reconduite, 2008, en français)

Car quoi qu'il arrive, seul Dieu sait :

"Donc je suis resté comme ça, dans la main de Dieu. Jusqu'à présent, je suis dans la main de Dieu. " (Madame Guéda D. à Dakar, Sénégal, propos recueilli par Douada Gary-Tounkara en français, en 2011)

Rester " dans la main de Dieu " : I'expression est on ne peut plus parlante, et l'est même sans doute exagérément pour un musulman devant se refuser à toute image de Dieu ${ }^{12}$. II n'en reste pas moins qu'elle est porteuse d'un sens très affirmé. Car cette main qui protège est aussi celle qui peut mettre en miettes, ou laisser choir. Rester dans la main de Dieu, c'est ainsi tout autant une menace qu'un privilège. L'amour de Dieu recèle un effroi, tout comme la peur de Dieu se nourrit d'un amour absolu. La question n'est donc pas tant celle de la peur ou du désir (de partir, de continuer), de la peur ou de l'amour (de Dieu), vu que l'une et l'autre vont de pair. Elle est bien plutôt celle de savoir, à un second degré, quel positionnement choisir de l'une à l'autre, quelle attitude emprunter avec, et depuis, chacun de ces sentiments. C'est une question de place et c'est une question de rapport.

"Chacun à la place où Dieu l'a posé ! Chacun à la place où Dieu l'a mis... Bon."

(Mamadou Bailo à Dakar, Sénégal, propos recueilli et traduit du wolof par Abdourahmane Seck, en 2012)

12 Elle est par çontre bien présente dans la Bible. Par exemple, dans l'Ancien Testament : "La main de l'Éternel n'est pas trop courte pour sauver " (Esaïe 59, 1), ou dans le Nouveau Testament : "Humiliez-vous donc sous la puissante main de Dieu " (1 Pierre 5, 6-7). 
Si chacun doit rester à sa place, alors il devient ardu de comprendre ceux qui prennent la route et viennent ainsi interférer dans les desseins du Créateur ceux qui ne se suffisent pas du sort qu'il leur a réservé, fût-ce celui de paysan pauvre attaché à sa terre et aux siens. La question de la place à tenir, à convoiter, à prendre est éminemment celle du rapport entretenu avec Dieu. Car si quitter son pays, se séparer de ses parents, de sa femme et de ses enfants, c'est ne pas se suffire de la place donnée par Lui, alors c'est aussi réfuter Son commandement, c'est s'immiscer dans Ses visées, c'est contester Sa puissance. C'est en somme en venir à un cas de conscience : une situation contradictoire à laquelle, évidemment, Dieu ne peut pas ne pas apporter la réponse ultime.

Car accepter tout de Dieu, c'est ce dont font profession de foi les migrants entendus. Or, à considérer que la volonté de Dieu exige une parfaite obéissance, ne pas rester parmi les siens, partir pour une aventure venant briser la linéarité de la vie au pays, ce serait peut-être bien déjà rompre avec elle, la Grande Volonté, et ce serait ne pas tenir parole : ni aux siens, ni à soi, ni à Dieu. Chose honteuse et cause du plus grand déshonneur... à moins de reconnaître, dans la rupture du destin premier, celui de pauvre dans un pays pauvre, et donc dans la nécessité du départ, un renouveau de cette Volonté : la révélation faite à celui qui doit partir d'un destin plus glorieux - un destin qui se réalisera au prix d'une soumission parfaite au Créateur, conjurant tout conflit intérieur dès lors que tout geste et tout sentiment né de ce geste, toute tentative de parvenir à une autre existence, toute réussite ou tout échec, auront été l'œuvre de Sa toute-puissance.

"Il y a gars qui me disait, hier, il y a un gars qui me disait: "ll y a une route qui quitte à la Mauritanie jusqu'au Maroc", j'ai dit: "AH BON ?" Si c'est ça, moi je vais me préparer, ah ouais, je vais me préparer, parce que un homme, il n'y a pas deux fois la mort. La mort c'est une seule fois, c'est vrai faut mourir dans des bonnes conditions mais bon, il n'y a pas deux morts. La vie, il faut se débattre pour gagner. [...] Moi j'ai pas eu peur mais j'ai la crainte de Dieu, j'ai beaucoup la crainte, je sais pas si vous vous êtes croyante, je sais pas, mais j'ai la crainte de Dieu, souvent il y a des choses je fais pas, parce que tu as envie de faire ça de faire ça, tu as envie de voyager. Dieu merci, je remercie Dieu " (Atta Koko à Dakar, Sénégal, recueilli en français par moi-même 2011).

Les difficultés, si ce n'est les contradictions, sont toujours là et font valoir une inquiétude : une inquiétude à laquelle Dieu, quoi qu'il arrive, vient apporter le dernier $\operatorname{mot}^{13} \ldots$ la question, toujours entière, restant celle de ce qu'il arrive avant ce dernier mot dont on sait qu'il sera par principe celui de Dieu, et sachant que ce qui se passe advient précisément en regard à la puissance de la parole ultime divine.

"Il faut se débattre pour gagner ", dit Atta Koko. Il le faut pour ne pas mourir. II faut se débattre pour tenir la mort en respect - donc en passer par une forme d'oubli, d'absence à ce qu'elle est - tout autant qu'il le faut pour bien mourir donc tenir à une pleine lucidité vis-à-vis d'elle. Il faut concilier l'une et l'autre, absence et lucidité, et c'est une conciliation difficile, c'est même une tâche impossible, si ce n'est fictivement sous la forme de sa perpétuelle remise en jeu,

13 On retrouve ce même processus, allant même jusqu'à une justification miraculeuse de leur présence à l'étranger (c'est Dieu qui les appelle dans ces nouveaux lieux), au sein des discours des migrants pasteurs évangéliques à Bruxelles, rencontrés par Maskens (2014). 
par le perpétuel retour de la parole et des pourparlers ou tractations que cette parole suppose.

La confusion des verbes " battre " et " débattre ", pour autant qu'elle ne serait pas délibérée, n'est pas davantage anodine dans la bouche d'Atta Koko. Elle dit bien quel conflit la parole recouvre et combien l'adresse au Tout-Puissant constitue, sous l'aspect de la louange, et face à la douleur, face à une peur du pire à la mesure d'un fol espoir, autant le signe de l'impossible dénouement des contradictions de celui qui se soumet au destin qu'il force, qu'un don de soi conjuratoire à Dieu, une apostrophe et un appel à la clémence. L'engagement individuel, ou l'agentivité, résulte justement de cette incertitude-là. Car s'il s'agit de rien moins que redéfinir sa place dans le monde donc d'en modifier l'ordre, $s^{\prime} i l$ s'agit là de s'arranger de la puissance divine en la reconnaissant comme la grande ordonnatrice de la rupture, il s'agit en retour, et avec la même gravité, d'en accepter les conséquences, c'est-à-dire de se soumettre en totalité à ce nouveau destin dont le départ, l'arrachement à la famille et au pays, détermine les conditions :

"C'est dur, mais c'est ainsi la vie. Il faut gagner sa vie. Dieu nous a créés pour ça. Tu vois dans ce monde-là il y a deux seules choses pour lesquelles nous sommes ici. Deux seules choses! Pour sa vie et de quoi vivre, donc vaquer à sa survivance et aussi veiller à ton sort à ta mort. II n'y a pas plus que ces deux choses. Toutes ces choses et objets autour n'ont aucune importance. " (Mouhamadou B. à Dakar, Sénégal, propos recueilli et traduit du wolof par Abdourahmane Seck, en 2012)

\section{Mission et soumission}

La parole des aventuriers se caractérise avant tout par des récits constitués de tous les éléments propres à une mythologie très ancienne en Afrique de l'Ouest, celle du récit d'aventure, incluant le conte et les récits guerriers : le personnage principal, héros quelle que soit l'issue de sa quête, retrace les étapes cruciales de son parcours fait d'obstacles physiques (le désert, la faim, la soif, la perdition en mer, etc.) et d'épreuves morales (le racisme, les outrages, la solitude, etc.), de personnages funestes comme les passeurs, les douaniers ou les policiers (ceux qui volent, humilient, frappent et souvent tuent) et d'adjuvants bénéfiques (ceux qui aident, orientent, nourrissent, sauvent, etc.) et de moyens pour parvenir à son but (la marche, les moyens de transport). Masqué par la dualité du départ à la fois espérance et rupture, faute d'orgueil et trait de bravoure, le motif du voyage est toujours identique : aller ailleurs (souvent en Europe), faire fortune, réussir, mais surtout ne revenir qu'une fois cette réussite concrétisée. L'important, comme je l'ai déjà écrit (Canut, 2014b) réside dans la possibilité de faire de cette aventure son propre récit, en singularisant un parcours migratoire tout en s'inscrivant dans la grande histoire des aventuriers, le panthéon des héros de l'aventure.

Cette inscription dans l'histoire des récits est souvent rappelée, notamment au Mali où la migration dans la région de Kayes est devenue au cours du $\mathrm{XXe}$ siècle une véritable institution liée aux politiques coloniales (Manchuelle, 1997 ; Timera, 2002 ; Jonsson, 2007 ; Barou, 2000). Prolongeant les récits de déplacement de populations ou les récits de fondation, de chasse ou de guerre 
qui ont constitué l'Afrique de l'Ouest avant la colonisation, les récits de migration valorisent, dans un cadre mondialisé, un parcours individuel ou collectif dont l'enjeu est justement de pouvoir le raconter, d'en laisser une trace dans I'histoire. Transmises de génération en génération, ces histoires empruntent aux légendes et immortalisent leur héros. La question du nom qui résonnera dans tout un village comme synonyme de victoire (Canut, 2014b) témoigne de ce désir de ne plus être n'importe qui, de ne plus demeurer cet être assis, passif, qu'on était avant le départ :

"Parce que tant que je n'aurai pas dormi rien qu'un seul mois en Europe, je ne serai jamais tranquille. J'ai toujours le projet... S'il plaît à Dieu ! (Cheik Oumar Sangaré à Doila, Mali, Frontière reconduite, 2008, en français)

Rien ne peut arrêter un aventurier comme Cheik Oumar Sangaré, un entrepreneur qui a déjà tenté quatre fois la traversée et vendu tous ses biens, car rien ne peut ternir ni faire tomber ce qui est devenu son horizon. Rien, sauf Dieu, qui fera tomber l'aventurier qu'il est. L'aventure est aussi une affaire entre soi et Dieu, quelles qu'en soient les péripéties, quels qu'en soient les protagonistes.

L'emploi de la première personne du singulier est le plus fréquent dans les récits adressés aux chercheurs et oppose le sujet à des événements, à des personnages incidents, au monde dont les rigueurs ou les cruautés ne lui sont pas épargnées, sans pouvoir un seul instant le faire dévier de sa route. II est comme en mission, poussé par des impératifs qui l'investissent et se concentrent sur sa personne. II est comme élu, porté au devoir par des nécessités contrariant d'autres nécessités, celles de la fidélité aux siens, à son pays, à sa place native, qui avaient eu valeur de lois.

L'horizon qui se propose à lui, la récompense aux sacrifices consentis, c'est un autre monde, un ailleurs aux marges du descriptible :

"Si on entre chez vous, on a l'impression de sortir d'un monde, pour entrer dans un autre monde. On dirait même qu'on ne respire pas le même air !"

(Cheik Oumar Sangaré à Doila, Mali, Frontière reconduite, 2008, en français)

Respirer le même air, c'est le partage fondamental : c'est le degré minimal du communautaire. Ne pas le respirer, c'est rompre ce lien ultime, intime même, qui attachait à l'unité du monde, au même monde pour tous : c'est consacrer I'entrée dans autre chose, c'est quitter le domaine des hommes ordinaires, et c'est aussi douter du lien d'équivalence entre l'unité du monde et l'unicité du Tout-Puissant. L'imaginer, c'est se tenir à deux doigts de la pensée sacrilège. Car alors tout se passe comme si devenait possible un monde des hommes où ceux-ci ne sont plus ceux que Dieu fait naître, guide, et fait mourir : un monde des hommes qui sont plus que des hommes, vivant dans la quasi-métamorphose du monde ou de soi-même :

"Déjà, quand tu vois les lumières, tu as envie d'être envoyé là-bas. Qu'on t'expédie en France ou en Espagne, sans rien avoir à faire. Tu aimerais à ce moment te transformer en oiseau pour t'envoler. Voilà !" (Cheik Oumar Sangaré à Doila, Mali, Frontière reconduite, 2008, en français) 
Cheik Oumar Sangaré, sous un jour poétique, laisse affleurer cette espérance : ne plus être soi. Comme ne l'est peut-être pas non plus celui qui se lève pour quitter son pays, comme a mué sans doute celui qui ne pleure pas face à la mort atroce de son ami, de son frère. Car rien ne peut arrêter l'aventurier dans sa traversée du monde, rien ne peut l'arrêter dans sa quête. Dans notre film Frontière reconduite, Baba, expulsé de France, ne cesse de rappeler combien la "France ", ce " gros nom " comme il le dit lui-même, est l'objet, confinant au sacré, de cette quête :

"Ça, c'est devenu dans notre vie maintenant. Aller. Va-et-vient, là. C'est notre vie maintenant, tu vois. II faut que j'aille. Même si j'ai... Un jour, j'ai parlé à ma femme. J'ai dit: "Mais. Si le visa... Vraiment, si je sais que je trouve pas de visa, je vais passer par l'Espagne" [...]. Voilà. Mais il y a des cinq- quinzaines de volontaires qui sont morts comme ça. Tout ça, c'est pour aller à la France. Tout ça, c'est ça. La France, c'est vraiment... On peut pas être sans aller là-bas. " (Baba, Tringa Marena,

Mali, Frontière reconduite, 2008, en français)

"On ne peut pas être sans aller là-bas ": cette phrase, en forme de précepte, met en rapport et même en équivalence le fait d'être, l'être (une valeur philosophique) et le fait d'aller, le déplacement (qui n'est pas une valeur philosophique, mais est beaucoup plus prosaïque). C'est comme un rapport du divin - du sacré - au profane : être, c'est exister par Dieu, quand aller c'est comme exister en dehors de lui. Et la tension se crée dans cette rencontre d'un désir profane, terrestre, et d'une injonction divine appelant à se soumettre à la loi du destin. Le point de résolution, quand la tension est trop forte, s'appelle la providence, ou l'intervention divine : elle peut revêtir l'aspect de la mort, pour une délivrance définitive. C'est pour cela qu'il faut l'accepter, ne pas chercher son sens : elle n'en a qu'un, et il est antérieur à sa survenue. C'est un fait accompli que le destin, devant lequel chacun est mis. La chose est entendue dès lors que ce sens est de tout temps celui de la grande justice, irrévocable par essence.

Ce " là-bas ", qui est presque un au-delà, qui pourrait être vu comme l'autre côté du miroir du monde, est une condition à l'existence : une existence pleine, et même inouïe, mais au risque de la mort : au risque de voir sur soi se refermer la main de Dieu - au risque aussi, pire sans doute, de voir cette main s'ouvrir et se retourner, laissant déchoir pour l'éternité I'aventurier trop ambitieux, trop effronté, l'abandonnant parmi les affres de l'oubli, de la nuit éternelle, loin, si loin du " gros nom " qu'il avait dû vouloir aussi pour lui.

\section{" Ceux qui meurent à cause de ce qu'ils désirent "}

Le "là-bas ", I'au-delà du monde, est aussi et surtout l'au-delà du sort commun réservé aux hommes sans aventure(s). $\mathrm{Si}$ " on ne peut pas être sans aller là-bas ", on ne peut pas davantage exister sans savoir... Mais sans savoir quoi ? Là est la question :

"NON, non non non non, c'est pas pour aller chercher de l'argent seulement. Parce que L'AVENTURE ça rapporte beaucoup de choses! Tu vas prendre de la connaissance,

tu vas apprendre, tu vas voir quelque chose que t'as jamais vu. Donc, soit l'argent, ça c'est l'objectif d'accord, tu vas travailler on te paye, mais, tu apprendras quelque chose que tu n'as jamais appris, tu verras quelque chose que t'as jamais vu. " (Baba, discussion en français avec Gabriela Pujol à Paris - Pujol, 2011) 
La réponse est donnée : il s'agit d'apprendre quelque chose qu'on n'a jamais appris. Curieuse formulation, en vérité, en forme de lapalissade : qui apprendrait quelque chose qu'il connaît déjà ? II est néanmoins possible d'y voir une intention non explicitée de rendre le caractère inouï de ce qui peut s'apprendre par l'aventure : I'affirmation selon laquelle ce qui s'apprend par l'aventure est en dehors du sens commun, au-delà de la mesure humaine, et se présente en tant que n'ayant pas été appris pour la raison qu'il n'aurait pas pu l'être - un quelque chose ayant rapport au fabuleux, dont l'imagination même illusionne sur l'apparence.

"La différence est telle que tu ne peux pas la raconter. Tu ne peux pas la décrire. La différence est très grande. "I n'y a pas de comparaisons. " (Cheik Oumar Sangaré à Doila, Mali, Frontière reconduite, 2008, en français)

Ce quelque chose de l'au-delà, tenu dans ce "là-bas " qui le met hors de portée des sens et de la pensée, ne peut qu'être sous-estimé. II ne peut être a priori que mal décrit, ou dessiné en creux. II échappe aux mots à mesure que les mots tentent de l'approcher. II échappe au langage à mesure qu'il rejoint le mythe. Et s'il est si mal formulable ou si difficilement descriptible, s'il ne se donne qu'en creux sous l'aspect de sa non-représentation, c'est qu'il est de l'ordre du surnaturel et tutoie le divin. Et de la même manière qu'il ressortit à une espèce de troisième lieu, de troisième monde, hors de l'enfer et hors du paradis, à la jointure entre terre et ciel, c'est qu'il se conçoit comme le lieu justifiant l'épreuve de sa quête, où un aventurier se soumet à l'expérience de sa propre révélation - l'expérience par laquelle il devient un autre homme, ou du moins plus que l'homme qu'il était : il devient quelqu'un qu'il ne connaissait pas.

Si les aventuriers ne se montrent que peu loquaces sur la mort, si elle paraît ne pas devoir les faire renoncer à leur aventure, ils n'en sont pas pour autant, loin de là, des enragés insensibles ou des fous inconscients comme il serait trop facile de vouloir les décrire.

« M : Pleurer? Eh! Ah non, pleurer...

$\mathrm{F}:$ Ton âme est sur l'argent. Tu n'as pas d'argent.

$\mathrm{M}$ : Tu penses à ça depuis la maison!

F : Tu ne pleures pas, tu suis Dieu. Tu t'endurcis.

$\mathrm{M}$ : En tout cas, sur la route, il n'y a pas de petite chose facile.

$\mathrm{F}$ : Tu dors par terre, tu dors dans la brousse. Certains meurent, certains sont malades, il

n'y a pas de médicaments, il n'y a rien. Ce qui nous pousse à faire cela, c'est la difficulté. " (Mariko et Fomba, avec Koyan à Doila, Mali, Frontière reconduite, 2008, traduit du bambara par moi-même)

Si la mort ne peut arrêter Mariko ou Fomba, ce n'est pas qu'ils y soient insensibles, ce n'est pas qu'ils s'apparenteraient à des indigènes ignorant jusqu'à la peur du néant, près de l'animalité que volontiers leur accole le primitivisme décomplexé sur l'autre rive de la Méditerranée. C'est d'abord qu'ils n'en ont pas le temps ni les moyens, et que l'aventure ne connaît qu'un répit, celui de l'éternité du trépas. Mais c'est également, et de surcroît, que placée sous le signe du Tout-Puissant, I'aventure cesse avec la mort comme elle serait rompue avec la rigueur, l'intransigeance et le dépouillement d'un verdict. L'épreuve est ordalique : elle ne permet aucun recours, elle ne suppose aucune défense. Elle 
ramène, alors que cet aventurier s'engage sur la voie d'un phénoménal accomplissement, alors qu'il déroge à la nécessité première de rester auprès des siens, alors qu'il paraît s'abandonner à la séduction d'un " là-bas " mythifié, elle ramène, cette épreuve, à I'humilité de ceux qui ne sont que matière et mémoire. Elle ramène à la parole : à ces mots infinis dont la mort est indemne - à la confession par les héros de l'aventure à la fois mortifiés et glorieux, honorables et communs, uniques et oubliés, de leur condition d'êtres humains qui n'en auront jamais fini avec le jugement de Dieu :

« En fait, on n'était rien que des aventuriers... Ceux qui meurent à cause de ce qu'ils désirent... C'était notre cas. " (Cheik Oumar Sangaré à Doila, Mali, Frontière reconduite, 2008, en français)

\section{Appendice}

Entrer dans l'épaisseur des mots, des expressions, du sens donné par les aventuriers à leur expérience suppose de concevoir le langage non pas comme un outil transparent, mais comme une pratique sociale qui engage celui qui parle à agir dans l'espace et le temps (Canut et Von Münchow, 2015). Si parler est essentiel tout au long du voyage, les aventuriers ne parlent pas de tout. Et surtout, ils n'en parlent pas de n'importe quelle manière. L'intérêt consiste à repérer les processus qui traversent l'ensemble des discours (qu'ils soient tenus à des proches ou à des étrangers, au départ, à l'arrivée ou au cours des trajectoires). Un des processus qui émergent fortement ici correspond ce que Orlandi (1994/1996: 59) nomme le " silence constitutif " repris par Von Münchow (2016) pour traiter des " non-dits ". La mise en silence (" I'ensilencement ", dirait Orlandi) de la mort par la plupart des migrants explique pourquoi, lorsqu'un chercheur sollicite des discours à son propos, il est nécessaire pour les interlocuteurs à la fois d'affirmer le caractère subsidiaire de la mort tout en référant systématiquement à son inscription religieuse : cette apparente contradiction, comme bien d'autres, ne cesse de parcourir les récits. Il serait toutefois erroné de penser que la parole s'oppose aux expériences vécues dont le chercheur peut rendre compte lors des ethnographies, celles qui indiquent par exemple l'absence de toute dimension sensible du rapport à la mort. La sociolinguistique (ou encore l'anthropologie du langage) en ce qu'elle s'inscrit dans un rapport permanent à l'ethnographie menée avec les aventuriers, permet au contraire la mise au jour des indexicalisations (Silvertein, 2003), des rapports, des analogies, entre la parole et les actes, et permet aussi de mettre au jour la performativité de la parole elle-même. Voilà pourquoi la dimension religieuse trop souvent ignorée dans les analyses des pratiques migratoires apparaît, avec le temps, fondamentale dans les discussions et les récits.

Loin d'une indifférence à la mort, la nécessité de ne pas en parler directement, de ne pas la commenter, tout en la rendant palpable à tout moment par l'évocation de Dieu, résulte de logiques particulières non pas liées à une quelconque spécificité (essentialisante) des migrants eux-mêmes, mais à des positionnements socio-subjectifs déterminés par la dimension (collective) religieuse au sein de laquelle l'action individuelle s'inscrit. Si Timera (2008) a été un des premiers à rendre compte de la place prépondérante de la religion musulmane comme moyen d'échapper à une perpétuation des formes de domi- 
nation coloniale, mais aussi de sa mise en scène permanente dans les relations à autrui tout au long des parcours migratoires (Ibid., 2009), les récits étudiés ici viennent à l'appui de ses analyses. Précisons toutefois qu'il n'y a pas, au fond, de contradiction entre cette apparente soumission à la volonté divine et l'impératif de dépassement de soi au niveau individuel. Les deux dimensions sont enchâssées l'une l'autre au point de pouvoir les penser interdépendantes : le surinvestissement dans l'action, qui fait du migrant un sujet actif à part entière, vient pallier l'affront fait au Tout-Puissant de contrer Sa volonté en dérogeant à la place octroyée, de donner l'impression de vouloir intervenir dans Ses affaires en forçant le destin. Ce que nous donnent à entendre les migrants, acteurs de leur existence, c'est bien la complexité du sens donné à leur vécu, de manière singulière dans chaque interaction. Loin des représentations visuelles émotionnelles, et parfois même sensationnelles des médias ou des politiques obnubilés par la mort des migrants, comme objet moral (alors qu'elle devient une vulgaire donnée statistique érigée en critère des programmes de lutte contre la migration) l'écoute et l'interprétation des paroles des aventuriers, voyageurs et réfugiés, dans toute leur ampleur, supposent au contraire de considérer chaque sujet parlant dans sa singularité, par le biais de ses mots, afin de comprendre la pluralité des significations données au parcours migratoire. De cette pluralité, le sociolinguiste a pour tâche de faire émerger les processus discursifs qui conditionnent les imaginaires, les dires et les actes : si la mort est décrite comme un banal accident de parcours possible ce n'est pas au nom d'un désintérêt pour elle, mais parce que le parcours lui-même se définit comme un combat permanent pour la réussite, quel qu'en soit le prix : arriver au point final afin de se réconcilier avec Dieu et de pouvoir affirmer, rétrospectivement, que partir était bien un " destin ", et le sien. 


\section{Références bibliographiques}

Akesson Lisa (2004) Making a Life, meanings of migration in Cape Verde, Phd thesis, Department of Social Anthropology, University of Gothenburg.

Andersson Ruben (2014) Illegality, Inc. Clandestine Migration and the Business of Bordering Europe, Berkeley, University of California Press, 416 p.

Apparadurai Arjun (1996) Modernity at Large: Cultural Dimensions of Globalization, Minneapolis, University of Minnesota Press, 231 p.

Bakhtine Mikhaïl (1952/1979/1984) Les genres du discours, in Mikhaïl Bakhtine, Esthétique de la création verbale, Paris, Gallimard, pp. 265-308.

Barou Jacques (2000) Migrations et travaux forcés en Afrique subsaharienne à I'époque coloniale, Hommes et migrations, 1228, pp. 51-61.

Bredeloup Sylvie (2014) Migrations d'aventures, Terrains africains, Paris, éditions du CTHS, $141 \mathrm{p}$.

Bredeloup Sylvie (2008) L'aventurier, une figure de la migration africaine, Cahiers internationaux de sociologie, 125, Paris, PUF, pp. 281-306.

Canut Cécile (2016) Migrants et réfugiés : quand dire, c'est faire la politique migratoire, Vacarme, [en ligne] consulté le 12/06/2016. URL : http://www. vacarme.org/article2901.html

Canut Cécile (2015) Paroles de femmes en colère (Santiago, Cap-Vert), Matérialités langagières et praxis sociales, in Cécile Canut et Patricia Von Münchow Éds., Le Langage en sciences sociales, Limoges, éditions Lambert Lucas, pp. 53-68.

Canut Cécile (2014a) L'Île des femmes [llia di mudjer], Paris, film produit parTutti quanti films, Ceped, ANR Miprimo, 53'.

Canut Cécile (2014b) On m'appelle le voyageur, in Cécile Canut et Catherine Mazauric Éds., La Migration prise aux mots. Mises en récits et en images des migrations transafricaines, Paris, Le Cavalier Bleu, pp. 261-278.

Canut Cécile (2013) Dans la frontière, Horizons Maghrébins, 68, pp. 79-95.

Canut Cécile (2008a) Frontière reconduite, Paris, film produit par Tutti quanti films, $1 \mathrm{~h} 30$ '.

Canut Cécile (2008b) Escale Tringa Marena, Paris, film produit par Tutti quanti films, 19 '.

Canut Cécile, Danos Felix, Him-Aquili Manon et Panis Caroline (à paraître) Le langage : une pratique sociale, Éléments d'une sociolinguistique politique.

Canut Cécile et Ramos Elsa (Éds.) (2014) Carnet de route d'un voyageur en Afrique de l'Ouest, Paris, Le Cavalier bleu, $96 \mathrm{p}$.

Canut Cécile et Sow Alioune (2014) "Nous nous appelons les voyageurs ". Mise en scène des parcours migratoires dans le théâtre des réfugiés d'Afrique centrale à Bamako, Cahiers d'études africaines, 213-214, pp. 383-414.

Canut Cécile et Von Münchow Patricia (2015) Le langage en sciences humaines et sociales, Limoges, Lambert Lucas, 212 p.

Choplin Armelle et Lombard Jérôme (2010) Suivre la route. Mobilités et échanges entre Mali, Mauritanie et Sénégal, EchoGéo, 14, pp. 1-24. 
Clifford James (1997) Routes, travel and translation in the late twentieth century, Cambridge, Harvard University press, $408 \mathrm{p}$.

Jonsson Gunvor (2007) The mirage of migration: Migration Aspirations and Immobility In a Malian Soninke Village, Institute of Anthropology, University of Copenhagen, Master thesis, $106 \mathrm{p}$.

Khosravi Shahram (2007)The "illegal" traveller: an auto-ethnography of borders, Social Anthropology/Anthropologie Sociale, 15 (3), pp. 321-334.

Kobelinsky Carolina (2016) Les vies des morts de la migration, Plein droit, $109(2)$, pp. 6-9.

Maitilasso Annalisa (2014) "Raconte-moi ta migration ". L'entretien biographique entre construction ethnographique et autonomie d'un nouveau genre littéraire, Cahiers d'études africaines, 213-214, pp. 241-265.

Manchuelle François (1997) Willing Migrants. Soninke labor Diasporas, Athens, Ohio University Press, 371 p.

Maskens Maïté (2014) "C'est Dieu qui nous a voulus ici... " : récit de migration et engagement religieux des pasteurs et fidèles pentecôtistes euro-africains à Bruxelles, Cahiers d'études africaines, 213-214, pp. 341-362.

Pian Anaïk (2009) Aux nouvelles frontières de l'Europe. L'aventure incertaine des Sénégalais au Maroc, Paris, La dispute, 237 p.

Pujol Gabriela (2011) Paroles de migration. Circulation des discours sur la migration dans l'espace politique des sans-papiers, mémoire de Master 2, ss la dir. de Cécile Canut, Université Paris Descartes, 199 p.

Quiminal Catherine (1991) Gens d'ici, gens d'ailleurs (migration soninké et transformations dans la vallée du fleuve), Paris, Christian Bourgois, 223 p.

Silverstein Michael (2003) Indexical order and the dialectics of sociolinguistic life, Language and Communication, 23 (3-4), pp. 193-229.

Timera Mahamet (2009) Aventuriers ou orphelins de la migration internationale. Nouveaux et anciens migrants " subsahariens " au Maroc, Politique africaine, $115(3)$, pp. 175-195.

Timera Mahamet (2008) Faire et défaire les identités avec le religieux. Migrations, ethnicité, "race " et religion, Habilitation à Diriger des Recherches, Université Paris Diderot, $439 \mathrm{p}$.

Timera Mahamet (2002) Les migrations des jeunes Sahéliens : affirmation de soi et émancipation, Autrepart, 18, Les Jeunes, hantise de l'espace public dans les sociétés du sud, Paris, IRD, pp. 37-49.

Von Münchow Patricia (2016) Quand le non-dit n'est pas l'implicite : comment rendre visibles les silences dans le discours?, Signes, 17, [en ligne] consulté le 12/01/2017. URL : https://halshs.archives-ouvertes.fr/halshs-01419431 


\section{Cécile Canut}

\section{"Tu ne pleures pas, tu suis Dieu... " Les aventuriers et le spectre de la mort}

Si la mort sur la route tend à constituer un indicible ou un inénarrable, la dimension métaphysique et religieuse est essentielle pour saisir l'absence de pathos dans les récits concernant les scènes de violence et de mort auxquelles les voyageurs ont été exposés. Cet article se focalise sur la dimension existentielle qui émerge des formes langagières utilisées par les aventuriers (d'Afrique de l'Ouest) dans leurs récits, en mettant en lien les passages d'évocation de la mort et de la place de Dieu dans plusieurs corpus issus de terrains divers. À travers la construction des récits, les modalités discursives ou les formes de dialogisme, on observe que la mort revêt un caractère de subsidiarité dans le parcours mis en mots. Dans cette quête, la place de Dieu comme garant d'un devenir devient alors essentielle. Pris dans la contradiction entre une injonction divine appelant à se soumettre à la loi du destin et le désir incommensurable de se déplacer pour forcer ce destin, les aventuriers ne cessent d'expérimenter une tension dont le point de résolution ne peut revêtir que la délivrance définitive.

\section{"You don't cry, you follow God..." Adventurers and the Specter of Death}

If death on the road tends to constitute an unspeakable or an unutterable, the metaphysic and religious dimensions are essential to grasp the absence of pathos in the narratives concerning extreme scene of violence and death to which the travelers were exposed. This paper focus on the existential dimension which emerge from the linguistic forms used by the adventurers (of West Africa) in their narratives or discourses, by linking the passages of evocation of death and the place of God in the corpus studied. Through the construction of narratives, the discursive modalities, or the dialogical forms, we observe that death has the character of subsidiarity. In this quest, the role of God as a guarantor of a future is decisive. Caught in the contradiction between a divine injunction calling to submit to the law of destiny and the immeasurable desire to move to force this destiny, adventurers do not stop experiencing a tension whose resolution point can only assume the definitive issuance.

\section{"No lloras, sigues a Dios... " Los aventureros $y$ el espectro de la muerte}

Si la muerte en el camino tiende a constituir un indecible o un inenarrable, la dimensión metafísica y religiosa es esencial para entender la ausencia de pathos en los relatos respecto a las escenas de violencia y de muerte a que vivieron los viajeros. Este artículo se centra en la dimensión existencial que surge de las formas lingüísticas utilizadas por los aventureros (de África del Oeste) en sus relatos, relacionando los pasajes de evocación de la muerte y del puesto de Dios en varios cuerpos de varias etnografías. A través de la construcción de relatos, de modalidades discursivas o de formas de dialogismo, se observa que la muerte reviste un carácter de subsidiaridad. En esa búsqueda, el lugar de Dios como garante de un porvenir se vuelve esencial. Atrapados en la contradicción entre un mandato divino que llama a someterse a la ley del destino y el inconmensurable deseo de moverse para forzar este destino, los aventureros no dejan de experimentar una tensión cuyo punto de resolución sólo puede asumir la liberación final. 